\title{
LEAST AND MOST DISJOINT ROOT SETS FOR DAUBECHIES WAVELETS
}

\author{
Carl Taswell
}

\author{
Computational Toolsmiths, Stanford, CA 94309-9925
}

\begin{abstract}
A new set of wavelet filter families has been added to the systematized collection of Daubechies wavelets. This new set includes complex and real, orthogonal and biorthogonal, least and most disjoint families defined using constraints derived from the principle of separably disjoint root sets in the complex $z$-domain. All of the new families are considered to be constraint selected without a search and without any evaluation of filter properties such as time-domain regularity or frequency-domain selectivity. In contrast, the older families in the collection are considered to be search optimized for extremal properties. Some of the new families are demonstrated to be equivalent to some of the older families, thereby obviating the necessity for any search in their computation. A library that displays images of all filter families in the collection is available at <www.toolsmiths.com>.
\end{abstract}

\section{INTRODUCTION}

Daubechies wavelet filters with minimal length and maximal flatness can be readily computed via spectral factorization of a symmetric positive polynomial [1]. All of the complex orthogonal, real orthogonal, and real biorthogonal families of the Daubechies class computable by spectral factorization have been studied experimentally in the systematized collection developed by Taswell $[2,3,4,5,6,7]$ over a wide range of vanishing moment numbers and filter lengths.

In contrast, angular parameterization methods have usually been demonstrated for wavelets with only one vanishing moment (i.e., less than maximal flatness) and very short length filters [8,9] with the exception of [10]. But the latter only verified orthogonality and vanishing moment numbers for the filters and did not attempt any search through the angular parametrization space for filters with desirable properties.

These comments highlight the essential question in the development of an algorithm for the design of wavelet filters: How much computational effort (measured by time, flops, and complexity of implementation) should be expended in the construction of a wavelet filter possessing which properties over which range of filter lengths? A basic assumption inherent in the systematized collection of Daubechies wavelets [6] hypothesizes that the spectral factorization approach affords the most economical generation of wavelet filters with the best variety and combination of properties over the widest range of filter lengths.

The economy of the spectral factorization method in comparison with the angular parameterization method is achieved by the reduced size of the search space for the filter root codes [6] relative to that for the filter coefficient angles [8]. In [6], conjectures were

Email: taswell@toolsmiths.com; Tel/Fax: 650-323-4336/5779. made regarding schemes to enhance the efficiency of the combinatorial search used in the design algorithm. This report investigates the next step in the development of an efficient algorithm: Can the search be completely eliminated?

Section 2 clarifies the distinction between constraint selected and search optimized filter families, explains the principle underlying the least and most disjoint root sets, and defines the new filter families. Section 3 presents examples and summaries of results for all of the new filter families. Section 4 concludes that the search $c a n$ be eliminated for those search optimized filter families for which equivalence has been demonstrated with constraint selected filter families.

\section{METHODS}

\subsection{General Framework}

Consider a filter expressed as the complex $z$-domain polynomial $F(z)$ with corresponding vectors for the roots $\mathbf{z} \equiv\left[z_{j}\right] \in \mathcal{Z}$ and the coefficients $\mathbf{f} \equiv\left[f_{n}\right] \in \mathcal{F}$. Associated with $F(z)$, assume there exist three parameters, vector $\gamma \in \Gamma$, vector $\xi \in \Xi$, and scalar $\lambda \in \Lambda$, respectively, that index the filter for a defined family, specify the particular filter within a search space, and characterize its properties.

Applying this notation to the orthonormal Daubechies [1] and Rioul [11] wavelets, $\gamma \equiv\left[\gamma_{1}, \gamma_{2}\right]=[N, K]$ represents the number $K$ of vanishing moments for wavelet filters of length $N=2 K$ and $N>2 K$, respectively. For angle space methods [8] to generate orthonormal wavelets, $\boldsymbol{\xi}$ represents the set of angles that specifies $\mathbf{f}$ for $F(z)$. For binomial space methods [6] to generate Daubechies wavelets, $\boldsymbol{\xi}$ represents the set of binary codes that specifies $\mathbf{z}$ for $F(z)$. In both cases, $\lambda$ represents a criterion obtained from an individual property or a weighted combination of properties computed from $\mathbf{z}$ and/or $\mathbf{f}$ (such as the filter's time-domain regularity [12] and phase nonlinearity [6], et c.) that characterizes $F(z)$.

Thus, $\gamma$ and $\boldsymbol{\xi}$ determine $F(z)$ and then $F(z)$ determines $\lambda$ with the mapping of spaces $\Gamma \times \Xi \mapsto \mathcal{F} \times \mathcal{Z} \mapsto \Lambda$. The parameters $\gamma$ and $\boldsymbol{\xi}$ that determine $F(z)$ are called the indexing parameter and specification parameter, respectively. The parameter $\lambda$ that is determined by $F(z)$ is called the characterization parameter. If $\lambda$ represents an individual property (rather than weighted combination of properties), then $\lambda$ is also termed a characteristic property of $F(z)$.

\subsection{Existence and Uniqueness}

Given a defined filter family indexed by $\gamma$, assume for fixed $\gamma$ that a finite sequence of filters $F_{i}(z)$ can be generated by and evaluated for corresponding sequences, respectively, of specification parameters $\boldsymbol{\xi}_{i}$ and characterization parameters $\lambda_{i}$. If $\Xi$ is an 
unbounded and/or continuous space, then it can be appropriately bounded and discretized to permit a countably finite sequence $\boldsymbol{\xi}_{i}$.

Assuming restriction to a countably finite space $\Xi$, then the corresponding spaces $\mathcal{F} \times \mathcal{Z}$ and $\Lambda$ are also countably finite. Further assuming a one-to-one invertible mapping and uniqueness of the elements $\lambda_{i} \in \Lambda$, then finite countability of unique elements for an invertible mapping implies that it is feasible to search for both elements $\underline{\lambda}=\min _{i} \lambda_{i}$ and $\bar{\lambda}=\max _{i} \lambda_{i}$ in the range and select the corresponding filters $F_{i}(z)$ in the domain.

\subsection{Definitions and Inferences}

A filter $F(z)$ is called extremal if it can be shown to possess a characterization parameter attaining an extreme manifested by either $\underline{\lambda}$ or $\bar{\lambda}$. A filter $F(z)$ is called search optimized if it is generated by an algorithm that optimizes $\lambda \in \Lambda$ with an exhaustive search to ensure identification of either $\lambda$ or $\bar{\lambda}$. A filter $F(z)$ is called constraint selected if it is generated by an algorithm that specifies sufficient constraints on $\boldsymbol{\xi}, \mathbf{f}$, or $\mathbf{z}$ to ensure uniqueness of $F(z)$ and selection of $F(z)$ without a search. An indexed set of filters $\left\{F_{\gamma}(z)\right\} \equiv\{F(z ; \gamma): \gamma \in \Gamma\}$ is called a family if all members of the set are generated by the same algorithm, a function $g(\boldsymbol{\xi} ; \boldsymbol{\gamma}), g(\mathbf{f} ; \boldsymbol{\gamma})$, or $g(\mathbf{z} ; \boldsymbol{\gamma})$, subject to the control of the indexing parameter $\gamma$.

Two different filter families $\left\{F_{\gamma}(z)\right\}$ and $\left\{F_{\gamma}^{\prime}(z)\right\}$ generated by two different algorithms $g(\cdot ; \gamma)$ and $g^{\prime}(\cdot ; \gamma)$ are $\mathcal{F}$-equivalent, or equivalent with respect to the filter coefficient space $\mathcal{F}$, if $\| \mathbf{f}_{\boldsymbol{\gamma}}-$ $\mathbf{f}_{\gamma}^{\prime} \|<\tau$ for all $\gamma \in \Gamma$ with given error tolerance $\tau(\mathcal{F})$. Analogously, $\left\{F_{\gamma}(z)\right\}$ and $\left\{F_{\gamma}^{\prime}(z)\right\}$ are $\mathcal{Z}$-equivalent, or equivalent w.r.t. the filter root space $\mathcal{Z}$, if $\left\|\mathbf{z}_{\boldsymbol{\gamma}}-\mathbf{z}_{\gamma}^{\prime}\right\|<\tau$ for all $\gamma \in \Gamma$ with given error tolerance $\tau(\mathcal{Z})$. Finally, they are $\Lambda$-equivalent, or equivalent w.r.t. the characterization parameter space $\Lambda$, if $\left|\lambda_{\gamma}-\lambda_{\gamma}^{\prime}\right|<\tau$ for all $\gamma \in \Gamma$ with given error tolerance $\tau(\Lambda)$.

A search optimized filter is necessarily an extremal filter, whereas a constraint selected filter may or may not be an extremal filter. If a constraint selected filter can be shown to be equivalent to a search optimized filter, then the constraint selected filter is also an extremal filter. $\mathcal{F}$-equivalence and $\mathcal{Z}$-equivalence of two filter families implies $\Lambda$-equivalence, but the converse is not true.

\subsection{Filter Families}

All filter families reported here are named, defined, and generated according to the conventions, notation, and methods established in [5, 6] for the systematized collection of wavelet filters computable by spectral factorization of the Daubechies polynomial. However, one of the families in [5, 6], DROLD, has been renamed DROMD in order to achieve consistency with the new collection of families introduced here: DCOMD, DCOLD, DROMD, DROLD, DRBMD, and DRBLD.

These acronyms abbreviate ' $D$ ' for Daubechies, ' $C$ ' and ' $R$ ' for complex and real, ' $\mathrm{O}$ ' and ' $\mathrm{B}$ ' for orthogonal and biorthogonal, and 'LD' and 'MD' for least and most disjoint. All of these filter families are constraint selected. They are compared here with other filter families from $[5,6]$ that are search optimized.

In addition to the factorization rules [6, Section 2.4.1] imposing the necessary contraints for complex orthogonality, real orthogonality, and real biorthogonality, the least and most disjoint families are defined according to constraints derived from the principle of separably disjoint root sets in the complex $z$-domain. Consider only the roots of the quotient filter

$$
Q_{\mathcal{D}}(z)=(z+1)^{-2(\mathcal{D}+1)} P_{\mathcal{D}}(z)
$$

where $P_{\mathcal{D}}(z)$ is the product filter and $\mathcal{D}$ is the degree of the Daubechies polynomial [6, Section 2.1.3]. For spectral factorization, split the set of roots from $Q(z)$ into two sets of roots $\left\{z_{k}^{a}\right\}$ and $\left\{z_{l}^{s}\right\}$ for the analysis and synthesis filters $A(z)$ and $S(z)$.

These root sets from $Q(z)$ must be disjoint with

$$
\emptyset=\left\{z_{k}^{a}\right\} \cap\left\{z_{l}^{s}\right\}
$$

(because common roots at $z=-1$ for both $A(z)$ and $S(z)$ from $P(z)$ have been excluded from consideration). Now let $\left\{\mathcal{C}_{i}^{a}\right\}$ and $\left\{\mathcal{C}_{j}^{s}\right\}$ denote finite collections of open convex regions with the largest area domains that do not intersect yet still cover the sets $\left\{z_{k}^{a}\right\}$ and $\left\{z_{l}^{s}\right\}$, respectively. More precisely,

$$
\begin{aligned}
\cup_{k} z_{k}^{a} & \subset \cup_{i} \mathcal{C}_{i}^{a} \\
\cup_{l} z_{l}^{s} & \subset \cup_{j} \mathcal{C}_{j}^{s} \\
\emptyset & =\cap_{i} \mathcal{C}_{i}^{a} \\
\emptyset & =\cap_{j} \mathcal{C}_{j}^{s} \\
\emptyset & =\left(\cup_{i} \mathcal{C}_{i}^{a}\right) \cap\left(\cup_{j} \mathcal{C}_{j}^{s}\right) .
\end{aligned}
$$

Finally, let $C$ denote the cardinality of the set

$$
\left\{\mathcal{C}_{i}^{a}: i=1, \ldots, I ; \mathcal{C}_{j}^{s}: j=1, \ldots, J\right\}
$$

as measured by the number $C=I+J$ of regions covering all the roots of $Q(z)$. Then root sets $\left\{z_{k}^{a}\right\}$ and $\left\{z_{l}^{s}\right\}$ are called least and most disjoint if $C$ is, respectively, the maximum or minimum possible subject to the constraints of the factorization rules imposed.

Spectral factorizations for the filter families designed with most disjoint roots $z_{j}=r_{j} e^{i \theta_{j}}$ can be summarized as follows: for DCOMD, $\left\{\left(z_{j}, z_{j}^{-1}\right):\left(r_{j}<1\right) \wedge\left(\theta_{j} \geq 0\right)\right\} \rightarrow A(z)$ and $\left\{\left(z_{j}, z_{j}^{-1}\right):\left(r_{j}>1\right) \wedge\left(\theta_{j} \leq 0\right)\right\} \rightarrow S(z)$; for DROMD, $\left\{\left(z_{j}, \bar{z}_{j}\right): r_{j}<1\right\} \rightarrow A(z)$ and $\left\{\left(z_{j}, \bar{z}_{j}\right): r_{j}>1\right\} \rightarrow S(z)$; for DRBMD, $\left\{\left(z_{j}, \bar{z}_{j}, z_{j}^{-1}, \bar{z}_{j}^{-1},\right): \theta_{j}<\theta^{*}\right\} \rightarrow A(z)$ and $\left\{\left(z_{j}, \bar{z}_{j}, z_{j}^{-1}, \bar{z}_{j}^{-1},\right): \theta_{j}>\theta^{*}\right\} \rightarrow S(z)$. The factorizations for the DCOLD, DROLD, and DRBLD filters designed with least disjoint root sets cannot be summarized as concisely. However, the corresponding algorithms order the roots by angle and impose the maximum number of alternations for the assignments in the split to $A(z)$ and $S(z)$. In addition for the biorthogonal case, the algorithm for DRBLD was modified further to devise another family called DRBRD with the letters 'RD' an abbreviation for regular disjoint.

\section{RESULTS}

Figure 1 displays spectral factorizations for each of the least and most disjoint filter families at $K_{a}=K_{s}=16$ for $\mathcal{D}=15$. Roots for $A(z)$ and $S(z)$ are marked with o's and x's, respectively. As an example of the principle of minimizing and maximizing $C$, observe that $C=3$ for DRBMD and $C=13$ for DRBLD. Note that $C \neq 2$ for DRBMD because convexity is required for each of the non-intersecting covering regions, and $C \neq 26$ for DRBLD because the largest area possible is required for each of the regions. Figure 2 displays the wavelets corresponding to $A(z)$ for the six examples in Figure 1. Both the real parts (solid lines) and imaginary parts (dotted lines) are shown for the complex wavelets. 
The constraint selected filter families introduced here were compared with the search optimized filter families from $[5,6]$ for $K_{a}=K_{s}=1, \ldots, 24$. Each member of the following sets of filter families was demonstrated to be $\mathcal{F}$-equivalent to the other members of the set with $\tau(\mathcal{F})$ at machine precision: \{DRBMD, DRBMU, DRBLS, DRBLR $\},\{$ DRBRD, DRBMR $\}$, DROMD, DROMA $\}$, and DCOMD, DCOMN $\}$. Additional figures available in [7] display values of various characteristic properties for the filter families. The families are listed in the legends sorted in order of the properties' median values for $A(z)$ over the range of the indexing parameter.

To assess $\Lambda$-equivalence of the families, these figures were examined visually and the corresponding numerical values in tables were checked for maximum deviation over the range $K_{a}=$ $K_{s}=1, \ldots, 24$. This evaluation revealed the following results: All of the orthogonal families are $\Lambda$-equivalent with $\tau(\Lambda)<0.2$ for time-domain regularity. The orthogonal pair $\{$ DROLD, DROLU $\}$ and biorthogonal pair $\{$ DRBMR, DRBLU $\}$ are each $\Lambda$-equivalent with regard to time-frequency uncertainty. The orthogonal pair $\{$ DROLD, DROLA $\}$ is $\Lambda$-equivalent with regard to phase nonlinearity. Note that since the pair $\{$ DRBRD, DRBMR $\}$ is $\mathcal{F}$ equivalent, then the pair $\{\mathrm{DRBRD}, \mathrm{DRBMR}\}$ is $\Lambda$-equivalent with regard to time-domain regularity and the pair $\{$ DRBRD, DRBLU $\}$ is $\Lambda$-equivalent with regard to time-frequency uncertainty.

\section{CONCLUSION}

Precise definitions have been introduced for the various components of a general filter design framework consisting of indexing parameters $\gamma \in \Gamma$, specification parameters $\boldsymbol{\xi} \in \Xi$, filter coefficients $\mathbf{f} \in \mathcal{F}$, filter roots $\mathbf{z} \in \mathcal{Z}$, characterization parameters $\lambda \in \Lambda$, their corresponding spaces, and the mappings between the spaces. Within this framework, definitions have also been introduced for filter families that are either search optimized or constraint selected and for their equivalence.

A new set of families was then introduced using the principle of least and most disjoint root sets measured by the number $C$ of nonintersecting open convex regions required to cover the root sets. The new set of constraint selected families includes the complex and real, orthogonal and biorthogonal, least and most disjoint families with acronyms DCOLD, DCOMD, DROLD, DROMD, DRBLD, and DRBMD, as well as an additional family with acronym DRBRD for biorthogonal regular disjoint. These families were evaluated for both $\mathcal{F}$-equivalence and $\Lambda$-equivalence with search optimized families from [6].

Several pairs of both kinds of equivalence were demonstrated for both orthogonal and biorthogonal families. If $\Lambda$-equivalence exists between a constraint selected family and a search optimized family with respect to a particular characterization parameter $\lambda$ as an extremal property, then the constraint selected family can be used to replace the search optimized family, and thus to obviate the necessity for a search in the computational algorithm. As an important example, the DROLD (least disjoint) family can be used as an effective substitute for the DROLA (least asymmetric) family.

The $\Lambda$-equivalent substitution of a constraint selected family for a search optimized family enables fast computation of those constraint selected family members for which the corresponding search optimized family members would require excessively slow computation. Because of the $\Lambda$-equivalence, this substitution can be performed without any loss greater than the tolerance $\tau(\Lambda)$ for the parameter $\lambda$ representing the characteristic property of the filter.

\section{REFERENCES}

[1] Ingrid Daubechies, "Orthonormal bases of compactly supported wavelets," Communications on Pure and Applied Mathematics, vol. 41, pp. 909-996, 1988.

[2] Carl Taswell, "Algorithms for the generation of Daubechies orthogonal least asymmetric wavelets and the computation of their Holder regularity," Tech. Rep., Scientific Computing and Computational Mathematics, Stanford University, Aug. 1995.

[3] Carl Taswell, "Computational algorithms for Daubechies least-asymmetric, symmetric, and most-symmetric wavelets," in Proceedings of the International Conference on Signal Processing Applications and Technology. Sept. 1997, pp. 1834-1838, Miller Freeman.

[4] Carl Taswell, The Systematized Collection of Wavelet Filters Computable by Spectral Factorization of the Daubechies Polynomial, Computational Toolsmiths, www . toolsmiths.com, 1997.

[5] Carl Taswell, "A spectral-factorization combinatorial-search algorithm unifying the systematized collection of Daubechies wavelets," in Proceedings of the IAAMSAD International Conference on Systems, Signals, Control, and Computers, Sept. 1998, Invited Paper and Session Keynote Lecture, Paper \#323, in press.

[6] Carl Taswell, "The systematized collection of Daubechies wavelets," Tech. Rep. CT-1998-06, Computational Toolsmiths, www.toolsmiths. com, June 1998.

[7] Carl Taswell, "Disjoint sets of Daubechies polynomial roots for generating wavelet filters with extremal properties," Tech. Rep. CT-1998-08, Computational Toolsmiths, www. toolsmiths. com, Aug. 1998.

[8] B. G. Sherlock and D. M. Monro, "On the space of orthonormal wavelets," IEEE Transactions on Signal Processing, vol. 46, no. 6, pp. 1716-1720, June 1998.

[9] S. Maslaković, I. R. Linscott, M. Oslick, and J. D. Twicken, "A library-based approach to design of smooth orthonormal wavelets," in Proceedings of the IEEE Digital Signal Processing Workshop, Aug. 1998, vol. 1, paper \#60.

[10] Carl Taswell, "Correction for the Sherlock-Monro algorithm for generating the space of real orthonormal wavelets," Tech. Rep. CT-1998-05, Computational Toolsmiths, www . toolsmiths.com, June 1998.

[11] Olivier Rioul and Pierre Duhamel, "A Remez exchange algorithm for orthonormal wavelets," vol. 41 , no. 8, pp. 550-560, Aug. 1994.

[12] Carl Taswell, "Empirical tests for the evaluation of multirate filter bank parameters," Tech. Rep. CT-1998-02, Computational Toolsmiths, www. toolsmiths. com, Mar. 1998. 


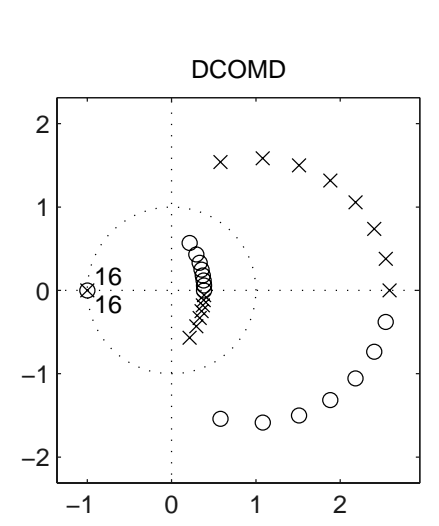

Disjoint Sets of Daubechies Polynomial Roots
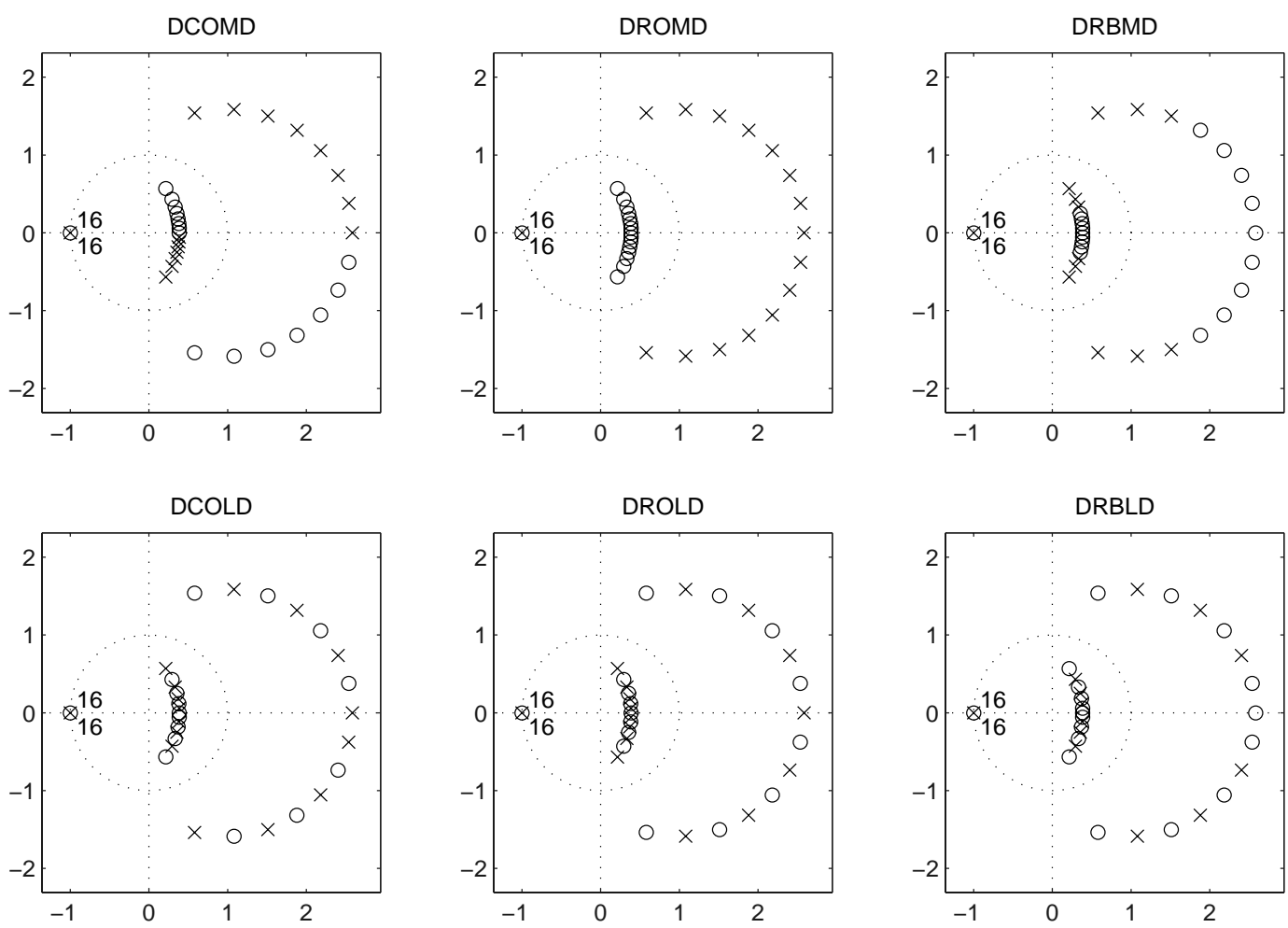

Figure 1: Examples of disjoint sets of Daubechies polynomial roots.

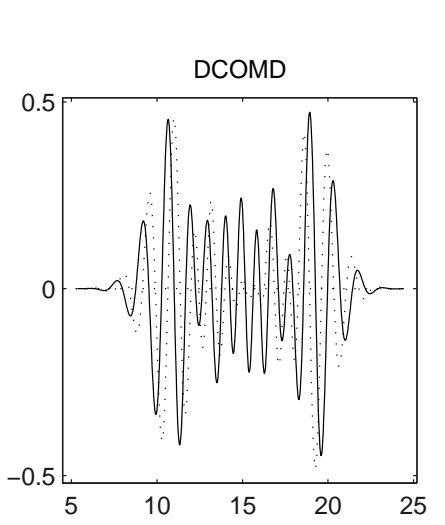

Wavelets for Disjoint Root Set Examples
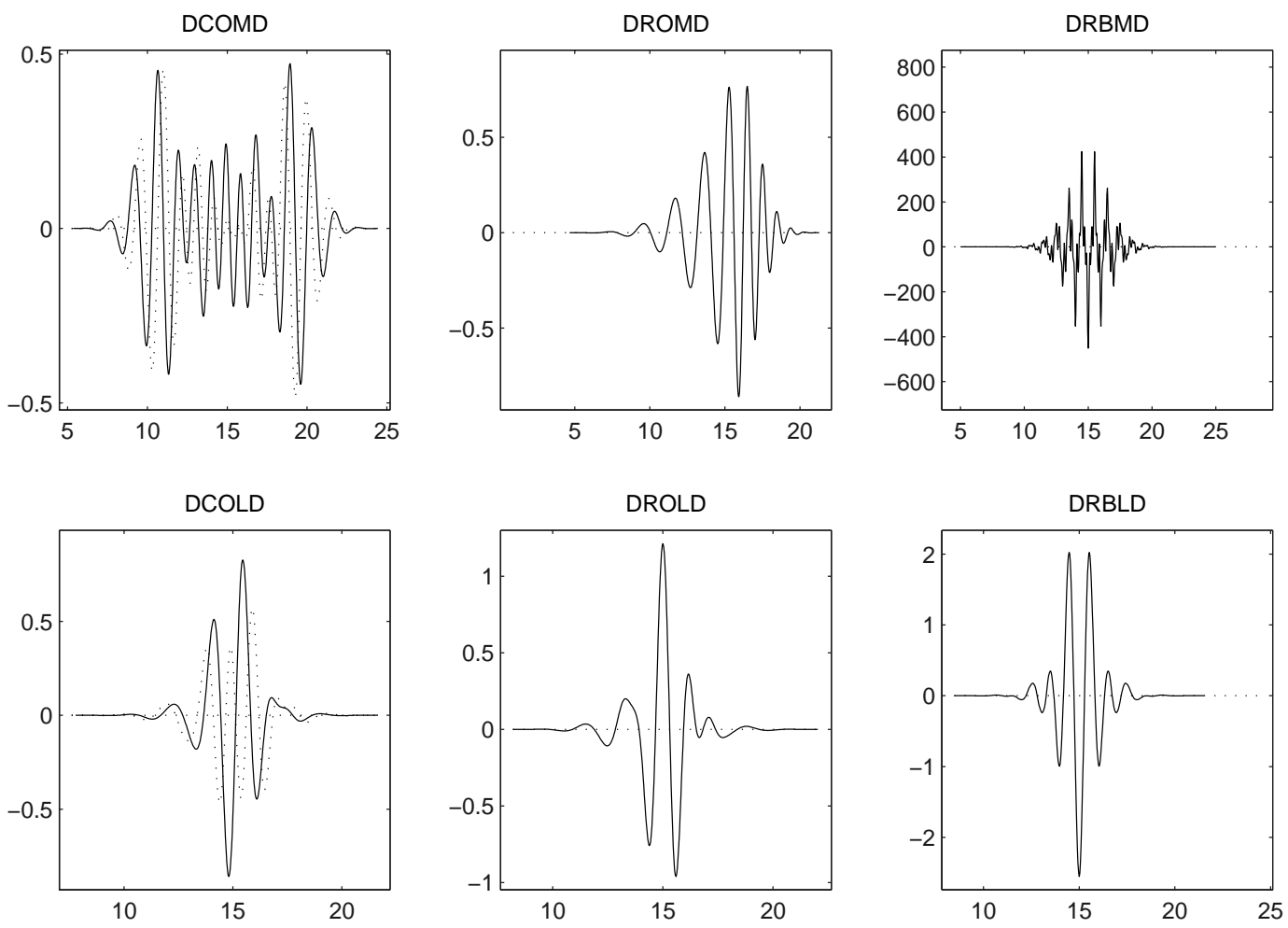

Figure 2: Analysis wavelets for disjoint root set examples. 